\title{
Interactive comment on "Changes in the future summer Mediterranean climate: contribution of teleconnections and local factors" by Monika J. Barcikowska et al.
}

\section{Anonymous Referee \#1}

Received and published: 4 March 2019

The paper tackles the problem of climate change in the Mediterranean, exploring the role of teleconnections and local feedbacks in modulating future projections. To this aim, climate simulations are designed and run using a state-of-the-art climate model. The authors find climate change in agreement with previous literature (warming and drying), however their findings suggest reduced amplitudes in change. They explain these differences with the improved ability of the model in simulating SNAO teleconnections and land surface feedbacks. 
literature, and in performing and discussing many analysis, but this makes the paper very long. My first general recommendation is to somehow shorten the manuscript, to facilitate the reader to be focused on the key messages delivered by the paper. For instance, discussions of previous findings is sometimes too detailed and redundant: accepted knowledge on summer climate in the Mediterranean region should be described in detail in the Introduction (or in a dedicated Background section) and briefly recalled when necessary in the text.

2. The main finding of the paper is the different amplitude of future projection of the Mediterranean climate simulated by the CM2.5 model in comparison with CMIP3 and CMIP5 simulations. To illustrate this crucial aspect, the authors refer to the existing literature on the topic. However, when quantitative differences are discussed, comparison with a Figure would be helpful. I suggest the authors to add some figures (in the supplement) showing projections of the future Mediterranean climate (precipitation and temperature) in the CMIP5 ensemble or, if downloading CMIP5 data is too time consuming, at least the CM2.1 model output, to show differences with the same model at lower resolution and not including the improved land model LM3.

3. Data: why NCEP reanalysis are selected for comparison? On the same period, ERAI data are available at higher resolution. ECMWF datasets are also available for the 20th century. Same question about precipitation data: why University of Delaware? Testing other temperature/precipitation datasets (CRU, EOBS) would change your results? In general, comparing your results with different datasets would improve the robustness of your conclusions.

4. One main issue of the paper is the choice of the time window to be analysed. The aim of the paper is to study summer Mediterranean climate, so that you select JJA. However, through the paper, different periods are selected for different analysis: JA or just July. I recommend to homogenise the period to be analysed (preferably JJA), for better comparison of the results. If there are specific reasons to analyse different period, these reasons should be highlighted.

ESDD

Interactive comment
Printer-friendly version

Discussion paper

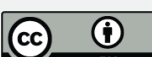


5. Methods section is rather long and sometimes confused: EOF analysis is described twice, the description of correlation/regression analysis is not really necessary here, as ESDD well as the reference to figures discussed later in the paper. I recommend to focus the section on the description of more sophisticated methods, such as EOF and stormtrack definition, and leave the description of correlation/regression analysis to the Results section. The section should be then shortened and optimised.

Interactive comment

6. Model validation: in Figures 1-4 you compare the CTRL simulation to the NCEP data, and I see some important biases in terms of intensity (SLP in monsoonal regions) and location of some features (axes of the anticyclonic circulations at $500 \mathrm{hPa}$ ). This is due to the fact that in NCEP reanalysis there is GHG forcing, which is not included in the CTRL simulation (as you also highlight in the text, P21, L34-37). It would not be more consistent to compare the CTRL simulation to a different period, i.e. a period of $20 \mathrm{C}$ reanalysis/precipitation less affected by GHG forcing?

7. SNAO simulation: the analysis of SNAO impact in Figure 5 is not compared with any reanalysis product. The SNAO impact on climate in Europe is explained with variations in the stormtrack (Figure 7): why reanalysis data are shown and not model simulation?

8. Figure 11: the caption of the figure and discussion at P17 should be improved. You first state that you compute EOFs for the CNTR simulation, than you project the HIST-PROJ fields onto the CTRL EOF to get the 1860-2100 time series. Than you state that you also compute EOFs separately for HIST and PROJ. Then you discuss the 1860-2100 time series in Fig. 11c, then you go to HIST and PROJ EOFs in Fig. $11 \mathrm{ab}$, and finally you discuss the contribution of the 1860-2100 SNAO to the end-ofcentury projection of precipitation (Fig. 11de). I find this discussion confusing. This is a crucial point of the paper and should be presented clearly. I recommend the authors to improve the readability of this section. Moreover, the discussion of the SNAO impact on temperature projection should be significantly expanded.

9. Results: the differences between CM2.5 and CMIP5 models in projecting the 
Mediterranean climate are explained with a) better representation of the SNAO teleconnection and b) improved representation of the land-atmosphere interaction by the ESDD LM3 model (see also the Abstract). However, the improvements of the LM3 model are not presented in the paper, nor how these new features actually improve the representation of the land-atmosphere interactions (e.g. representation of soil moisture, evapotranspiration, albedo). A brief presentation of the LM3 model as well as a discussion of how it improves climate simulation in the Mediterranean is needed.

10. Conclusions: most of the paper is devoted to the analysis of the SNAO teleconnection and its impact on future climate change in the Mediterranean, which show a significant (P17, Figure 11) impact on precipitation in southern Europe. And in the abstract you indicate this as one of the main results of the paper. Conversely, in the Conclusions you somehow reduce the importance of the SNAO impact (P22, L38-40), explaining the differences with the CMIP5 simulations as a consequence of the improved land model. This point needs to be clarified.

Minor comments

P2, L15: the connection between the Mediterranean and the African monsoon has been robustly described as Mediterranean $\rightarrow$ Africa (see papers by Raicich et al. 2003 and Rowell 2003 [https://doi.org/10.1175/15200442(2003)016<0849:TIOMSO>2.0.CO;2]). The influence of the African monsoon on the Mediterranean is less clear: Ziv et al. 2004, but also Fontaine et al. 2011 [https://doi.org/10.1002/joc.2108], actually find a link between convection in Africa and subsidence in the Mediterranean, however the mechanism is still not clear (see Gaetani et al. 2011 [https://doi.org/10.1029/2011GL047150]). Indeed, the Asian monsoon could be dominant in modulating the Mediterranean-Africa connection. Please modify the sentence to account for this aspect.

Printer-friendly version

P2, L26-29: please add a reference.

Discussion paper

P3, L18-20: please add a reference.

Interactive

comment

Discussion paper

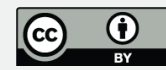


P3, L43: "fixed levels of radiative forcing", do you mean 'radiative forcing from fixed levels of emission/concentration'?

ESDD

P4, Methods: is the model fully coupled? How many vertical levels are in the ocean model?

P6, L15-16: this sentence should be moved to the Results section.

Interactive

comment

P6, L18-19: what do you mean with "vector time series"? The time series of the vector containing spatial data?

P7, L39: from Figure 4, precipitation magnitude is actually, not "apparently", larger than observations.

P7, L45: "none of the CMIP5..."

P8, L1: do you mean that the CM2.5 runs in the CMIP5 archive are better than other models in the archive? Or do you refer to the runs you analyse in this paper? If this is the case, you should provide a figure to support this statement.

P8, L10-13: when discussing the impact of NAO and SNAO on European climate, add references.

P8, L17: "and rather wet conditions".

P8, L18-19: add references on future projections of SNAO.

Section 3.2: the objective is to test the capability of the model in simulating the SNAO as an independent internally-generated mode of climate variability. However, the long introduction at P8-9 does not actually help in understanding why this is necessary. Is the internal variability modulated at multidecadal time scales? Is this modulation externally forced? Please try to clarify motivations and objectives of the section.

P8, L20-26: this paragraph is confusing: on the one hand, it is true that different approaches/datasets may lead to uncertainty in the observed SNAO-Mediterranean tele-
Printer-friendly version

Discussion paper 
connection; on the other hand, uncertainties in model simulations originate from model shortcomings. Therefore uncertainty in the real and model worlds could originate from both intrinsic non-linear nature of the phenomenon and inadequate statistical/modelling tools. Please rephrase.

P9, L8-9: I cannot understand why and how anthropogenic forcing should intensify SNAO contribution (to the summer atmospheric circulation over North Atlantic). Please explain.

P9, L34: add the figures for July and August to the Supplement.

P9, L35: what is the interest of comparing with the HadCM3 model?

P9, L42: is it HadGEM1 or HadCM3?

P13, L6-7: why an East Mediterranean index is used to compute correlation in Figure $8 \mathrm{~d}$, instead of the first EOF for NCEP omega?

P14, L25-26: what do you mean with "estimated at the original model resolution"? Do you mean "computed"?

P14, L32: east.

P17, L1-3: I don't understand why you refer to Fig. 10a (showing end-of-century projections) to discuss changes in SNAO. You could maybe use this figure to support your analysis of future SNAO.

P17, L14-16: it would be preferable to present the regression method to estimate the SNAO impact here rather than in the Method section.

P17, L43: "warming is lower over ... than ..."

P18, L7-12: it is not clear to me whether you are discussing you results (in Figure 10) or previous findings. If you discuss your results, please add more references to Figure 10 , otherwise add a reference to a paper.
ESDD

Interactive

comment
Printer-friendly version

Discussion paper 
P20, L39-41 and 42-45: please add references.

P21, L20: "preindustrial value".

ESDD

P22, L9-10: please add a citation to CMIP5 results.

Figures: for better comparison, figures presenting climate change in the Mediterranean

Interactive should share the same geographical boundaries. Same recommendation for figures comment presenting SNAO and Asian monsoon teleconnections, respectively.

Figure 6: what do contours represent? The sign looks reversed with respect to the standard SNAO pattern. Could you please fix this, not to mislead the reader?

Figure 7: does it make sense to project the SNAO index derived from 20CR onto NCEP data? Why not just analyse one dataset?

Figure 8: Do you perform EOF on omega 500 and 300 together? Or is EOF analysis performed separately on omega 500 and 300 ? If this is the case, which time series do you use for correlations?

Figure 10: wind is displayed at which level? Is not model resolution 0.5 ?

Figure 11: in panels $d$ and e you show regressions, while in Fig. 9c you show correlations.

Figure 12: is omega at 200 or 500 ? See P18, L23.

Supplement: please follow the logical order of the paper to number the figures. Also please write complete captions, avoiding to refer to captions in the main text.

Interactive comment on Earth Syst. Dynam. Discuss., https://doi.org/10.5194/esd-2018-85, 2019.

Printer-friendly version

Discussion paper 